\title{
How sample size can effect landslide size distribution
}

\author{
Langping $\mathrm{Li}^{1}$, Hengxing Lan ${ }^{1,2^{*}}$ and Yuming $\mathrm{Wu}^{1}$
}

\begin{abstract}
Background: Landslide size distribution is widely found to obey a negative power law with a rollover in the smaller size, and has been exploited by many researchers to inspect landside physics or to assess landslide erosion and landslide hazard. Yet, sample size has effect on the statistics of landslide size even though we manage to avoid complications associated with landslide datasets and statistical treatments.

Results: In this paper, a series of stochastic simulations were implemented to explicitly and systematically quantify the effect of sample size. The results show that, the errors of parameters estimated based on small sample size can be considerably large. For a sample size of 100, the relative error of the estimated landslide erosion rate that has a probability of $50 \%$ can approach $100 \%$. In addition, small sample size also obscures the statistical significance of the variances in parameters between different subsets of the same dataset. Although inconsistency was found regarding how the power exponent varies with rainfall intensity, numerical results suggest that the variance observed in a dataset with a small sample size may be not statistically significant.
\end{abstract}

Conclusions: This paper not only reveals the potential effect of sample size on exploiting landslide size distribution but also presents procedures for quantifying this issue in future studies.

Keywords: Landslide size distribution, Sample size, Statistical significance, Power law, Rollover

\section{Background}

The frequency of landslide is widely observed to decrease as a power law with the increase of size after a maximum value (Stark and Hovius 2001; Malamud et al. 2004; Brunetti et al. 2009). This partial power law behavior is unique because it is represented by not only a heavy tail as observed in many phenomena (Caers et al. 1999; Cheng 2008; Pinto et al. 2012; Kolyukhin and Tveranger, 2014), but also a rollover in the smaller size. The exponent of the power law tail $(\gamma)$ and the rollover $(R)$ are therefore the two most characteristic parameters. On one hand, landslide size distribution is crucial for quantitative analysis of landslide hazard (Hungr et al. 1999; Guzzetti et al. 2005) and earth surface processes

\footnotetext{
* Correspondence: lanhx@igsnrr.ac.cn

${ }^{1}$ State Key Laboratory of Resources and Environmental Information System, Institute of Geographic Sciences and Natural Resources Research, Chinese Academy of Sciences, 11A, Datun Road, Chaoyang District, Beijing 100101, China

${ }^{2}$ Department of Civil and Environmental Engineering, University of Alberta, Edmonton, Canada
}

(Hovius et al. 1997; Larsen and Montgomery 2012). On the other hand, the emergence of the power law tail and the rollover are mysteries that still lack widely accepted physical explanations (Pelletier et al. 1997; Katz and Aharonov 2006; Stark and Guzzetti 2009; Lehmann and Or 2012; Frattini and Crosta 2013; Alvioli et al. 2014; Li et al. 2014 and references therein). Therefore, landslide size distribution has been exploited by many researchers either to inspect the physics of landslides or to assess landslide erosion and landslide hazard.

The statistics of landslide size could be obscured by complications associated with landslide datasets and statistical treatments in the first place. The following strategies had been adopted to mitigate these complications: 1) using event-based rather than historical landslide datasets (Malamud et al. 2004; Ghosh et al. 2012); 2) using the same dataset prepared by the same author instead of datasets prepared by different authors (Iwahashi et al. 2003; Guzzetti et al. 2008; 
Chen 2009); and 3) using the maximum likelihood estimation (MLE) rather than linear regression to estimate both the power exponent and the rollover (Fiorucci et al. 2011; Ghosh et al. 2012). Nevertheless, even without these complications, limited sample size can also cast a shadow on the statistics of landslide size. Sample size effect is in fact a problem faced by many disciplines (Lazzeroni and Ray 2012). The error on the estimated scaling parameter of power law distributions from sample size effects has been investigated (Clauset et al. 2009). Yet, the potential effect of sample size on the statistics of landslide size has not been explicitly addressed. The fact that small differences in parameters of the size frequency relationship may produce huge mismatches in the derived landslide erosion rates (Korup et al. 2012) suggests the significance of this issue in some respects. This paper aims to quantitatively inspect the possible effect of sample size on exploiting landslide size distribution. We will focus on the landslide area distribution because so far no satisfactory distribution function for landslide volume has been proposed and most empirical datasets do not have landslide volume data.

\section{Methods}

\section{Landslide area distribution}

The widely adopted double Pareto function (Stark and Hovius 2001) and Inverse Gamma function (Malamud et al. 2004) were used to characterize the landslide area distributions. And parameters of the two distributions were estimated by the maximum likelihood estimation (MLE). It is inconclusive whether the two functions are mathematically and physically eligible to represent the landslide area distributions in the real world, but they are a practical choice since there are capable of characterizing both the power law tail and the rollover of landslide area distribution. To examine the assumption that landslide size distribution is characterized by a power law tail goes beyond the topic of this paper, we therefore did not test that hypothesis. For the same reason, we did not examine whether log-normal function (ten Brink et al. 2009; Mackey and Roering 2011), logarithmic function (Issler et al. 2005; Che et al. 2011) or exponential function (Montgomery et al. 1998) is an alternative to characterize the empirical data of landslide size. Analytical distribution function for landslide area yielded by maximizing Tsallis entropy (Chen et al. 2011) were not used as it is accompanied by complications (Li et al. 2012).

The expression of the double Pareto distribution is:

$$
p_{\mathrm{dp}}(A)=\frac{\beta}{t(1-\delta)} \frac{\left[1+(m / t)^{-\alpha}\right]^{\beta / \alpha}}{\left[1+(A / t)^{-\alpha}\right]^{1+\beta / \alpha}}(A / t)^{-\alpha-1}
$$

where

$$
\delta=\left[\frac{1+(m / t)^{-\alpha}}{1+(c / t)^{-\alpha}}\right]^{\beta / \alpha}
$$

$A$ is landslide area, $p_{\mathrm{dp}}(A)$ is probability density of landslide area, $\alpha, \beta$, and $t$ are constants, while $c$ and $m$ are two cutoffs define the interval within which the normalization condition satisfies. This function can be approximated by a negative power law (tail) with an exponent of $-\alpha-1$ for large size events and a positive power law with an exponent of $\beta-1$ for small size events separated by a maximum (rollover). With the maximum probability density, we have the rollover at area value:

$$
R_{\mathrm{dp}}^{A}=\exp \left[\frac{1}{\alpha}(\ln (\beta-1)-\ln (\alpha+1))+\ln t\right]
$$

We therefore do not use $t$ as the "crossover" of $p_{\mathrm{dp}}(A)$ as suggested by Stark and Hovius (2001), but use the area with maximum probability density as the "rollover". We chose 1 and $10^{10}$ as the two cutoffs to ensure all the landslide area values in empirical datasets fall into this scope and found similar choices (e.g., 1 and $10^{8}$ ) get almost the same estimates of parameters.

The expression of the Inverse Gamma distribution is:

$$
p_{\mathrm{ig}}(A)=\frac{1}{a \Gamma(\rho)}\left[\frac{a}{A-s}\right]^{\rho+1} \exp \left[-\frac{a}{A-S}\right]
$$

where $A$ is landslide area, $p_{\mathrm{ig}}(A)$ is probability density of landslide area, $\Gamma(\rho)$ is the gamma function of $\rho$, while $\rho$, $a$, and $s$ are constants. The normalization condition is satisfied within $[s,+\infty)$. This function can be approximated by a negative power law (tail) with an exponent of $-\rho-1$ for large size events and an exponential function for small size events separated by a maximum (rollover). With the maximum probability density, we have the rollover at area value:

$$
R_{\mathrm{ig}}^{A}=\frac{a}{\rho+1}+s
$$

\section{Average landslide volume}

The landslide volume $(V)$ is found to relate to landside area $(A)$ with a scaling exponent $\tau$ and an intercept $\varepsilon$ (Guzzetti et al. 2009; Larsen et al. 2010; Klar et al. 2011) such that:

$$
V(A)=\varepsilon A^{\tau}
$$

Then, an average landslide volume $\left(V_{\mathrm{a}}\right)$ can be defined and calculated by:

$$
V_{\mathrm{a}}=\int V(A) p(A) d A=\int \varepsilon A^{\tau} p(A) d A
$$

where $p(A)$ is either $p_{\mathrm{dp}}(A)$ or $p_{\mathrm{ig}}(A)$ in our study, and the range of integration corresponds to the interval 
within which the normalization condition satisfies. The average volume is important because it leads to the amount of landslide erosion and further the landslide erosion rate if the total landslide number is given. In other words, Eq. (6) makes it possible to inspect how the parameter estimation of landslide area distribution affects the estimation of landslide erosion rate. In our study, the relationship $V=2.59 A^{1.05}$ deduced from the Fujian historical landslide inventory ( $\mathrm{Li}$ et al. 2014) is used, and Eq. (y6) is calculated numerically.

\section{Stochastic simulation}

A straightforward procedure is designed to inspect the effect of sample size on the reliability of the parameter estimation of landslide size distribution. It is assumed that: 1) the theoretical distribution of landslide size with respect to a certain event within a certain area is constrained by physical factors and is therefore predetermined; 2) the number of landslides occurring in a certain event within a certain area is finite; and 3) the size of each individual occurred landslide is stochastic. So, we firstly introduce a predefined theoretical distribution of landslide area, and then draw $N$ values of area from the theoretical distribution using Monte Carlo simulation, where $N$ is the sample size. Values of sample size span from 100 to 10,000 and are logarithmic spaced. With regard to each sample size, 1,000 Monte Carlo samples are produced to reveal the stochasticity of the estimated parameters. If the sample size is large enough, the estimated parameters of the 1,000 Monte Carlo samples are expected to have a mean value similar to the parameters of the theoretical distribution and a low standard deviation. On the contrary, if the sample size is small, a mean value far different to the theoretical value and a high standard deviation are expected.

Similarly, we also use a straightforward way to inspect how the sample size influences the statistical significance of the comparison of the parameters of landslide size distribution between different subsets. Firstly, with regard to each sample size, the sample with parameters most similar to the theoretical values is picked out from the formerly produced 1,000 Monte Carlo samples as the test sample for this sample size. Then, for each sample size, the corresponding test sample is randomly subdivided into two subsets according to a subdividing ratio. And six subdividing ratios, namely $1: 1,2: 1,3: 1$, $4: 1,5: 1$, and $6: 1$, are used to inspect the effect of subdividing ratio as well. For each test sample, the random subdivision is repeated 1,000 times for each subdividing ratio. If we take "the observed differences in parameters between the two subsets are attributed to random processes" as the null hypothesis, the region of rejection and also the region of acceptance for a certain significance level (e.g., 0.05) can be estimated according to the statistics of the variances in parameters observed in the 1,000 random trials.

\section{Landslide dataset}

Heavy rainfall struck Fujian in the mid-to-late June, 2010 and induced large numbers of landslides. The XiayangWangtai ( $X W$ as an abbreviation) area, for which SPOT images with $2.5 \mathrm{~m}$ spatial resolution taken shortly after this rainfall event are available (Fig. 1), suffered greatly from this rainfall event. Rainfall records show that the cumulative rainfall in the $X W$ area in this period exceeds $300 \mathrm{~mm}$ and approaches $600 \mathrm{~mm}$ (Fig. 1a). Landslides in the study area were manually mapped on the SPOT images in a GIS platform. The bright brownish area representing both the failure and the deposition zones were visually delineated as fresh landslide areas (Fig. 1b). Field reconnaissance had helped to link real landslide scenarios with features on the SPOT images (Fig. 1c, d, e). In addition, the topography was inspected through a digital elevation model (DEM) with $5 \mathrm{~m}$ spatial resolution to support the mapping. The brownish area in gullies that represents the traces of debris flows and floods were not delineated. As seen during field surveys, shallow earth slides account for most of the landslides in the $X W$ area in this event. Totally 12,524 landslides were identified in the study area which means a density of about 43 landslides per square kilometer. We will refer to this inventory as " $X W$ dataset".

The high resolution of the SPOT images and DEM implies that this event-based landslide dataset ought to be substantially complete for landslides with area larger than $25 \mathrm{~m}^{2}$ regarding this rainfall event within this $X W$ area. In addition, only one interpreter was involved in the mapping procedures. This suggests that potential errors due to the diversity of the skills and experience of the interpreters are avoided in the following statistical analysis. Two subsets of the $X W$ dataset, namely $R 1$ and $R 2$, were produced according to the $450 \mathrm{~mm}$ cumulative rainfall isoline as shown in Fig. 1a. The probability distributions of landslide area fitted to the empirical datasets using MLE are shown in Fig. 2. The statistical parameters, including the estimated exponents of the power law tail $(\gamma)$ and the estimated rollovers $(R)$, are presented in Table 1 . In the following analysis, we will take the fitted landslide size distributions of the $X W$ dataset as the predefined theoretical distributions for the Monte Carlo simulations, and will also test whether the observed variances in parameters between the $R 1$ and $R 2$ subsets is statistically significant or not.

\section{Results}

The reliability of estimating parameters

The mean and standard deviation of the estimated parameters $(\gamma$ and $R$ ) with respect to each sample size are 


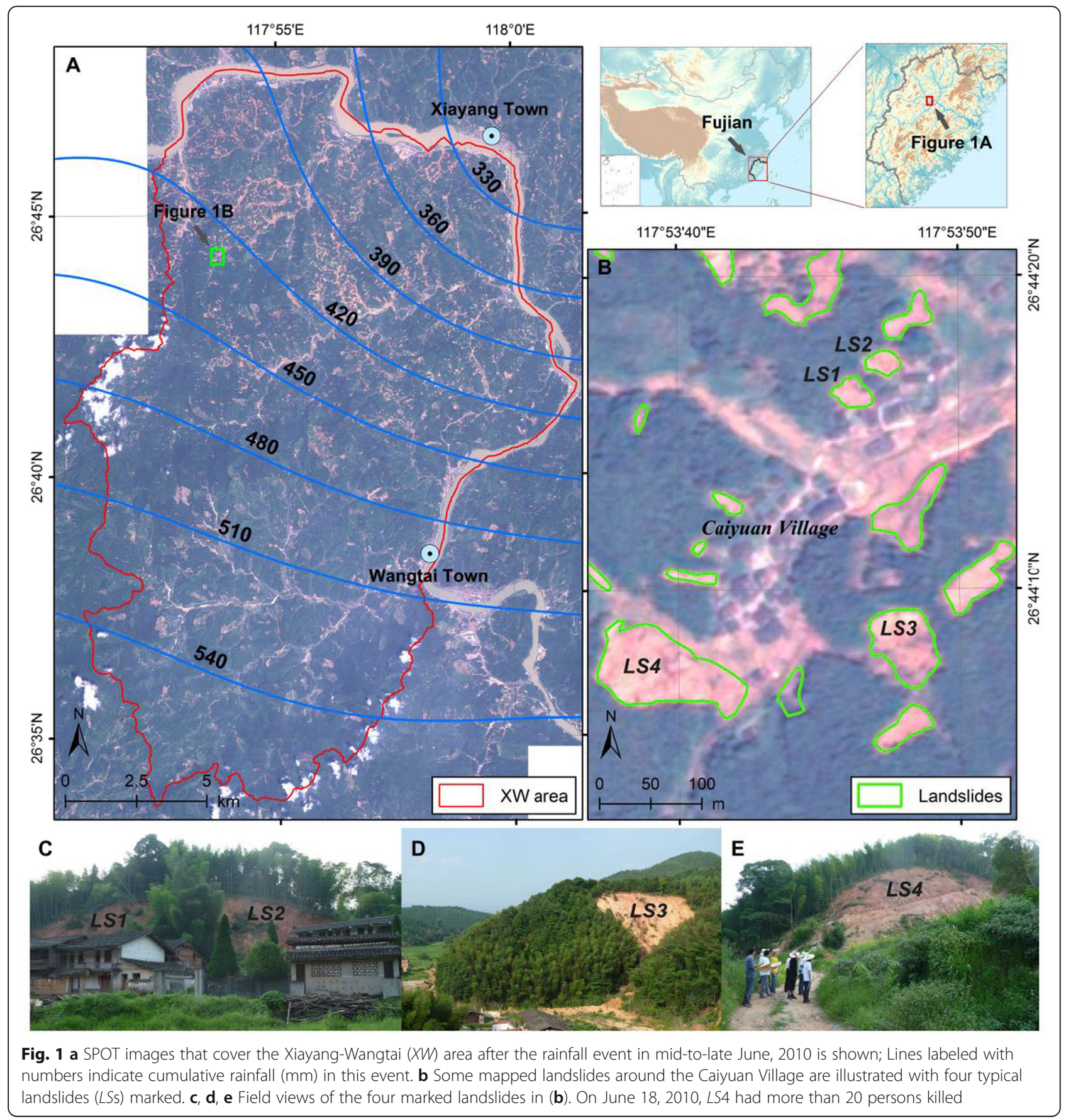

presented in Fig. 3. For both the double Pareto distribution and the Inverse Gamma distribution, as sample size gets smaller, the mean of the estimated parameters gets more deviated from the theoretical value (Fig. 3a, b) while the standard deviation gets larger (Fig. 3c, d). In addition, the probability that the estimated value has a relative error less than $5 \%$ is calculated to quantify the performance of parameter estimation. Not surprisingly, for both the exponent and the rollover, this probability decreases dramatically as sample size gets smaller (Fig. 3e, f). Compared with the Inverse Gamma distribution, the estimated exponents using the double Pareto distribution have lower standard deviations. Therefore, the performance of the double Pareto distribution on estimating the exponent is relatively better than the Inverse Gamma distribution (Fig. 3e). On the contrary, on estimating the rollover, the performance of the Inverse Gamma distribution is slightly better than the double Pareto distribution (Fig. 3f). 

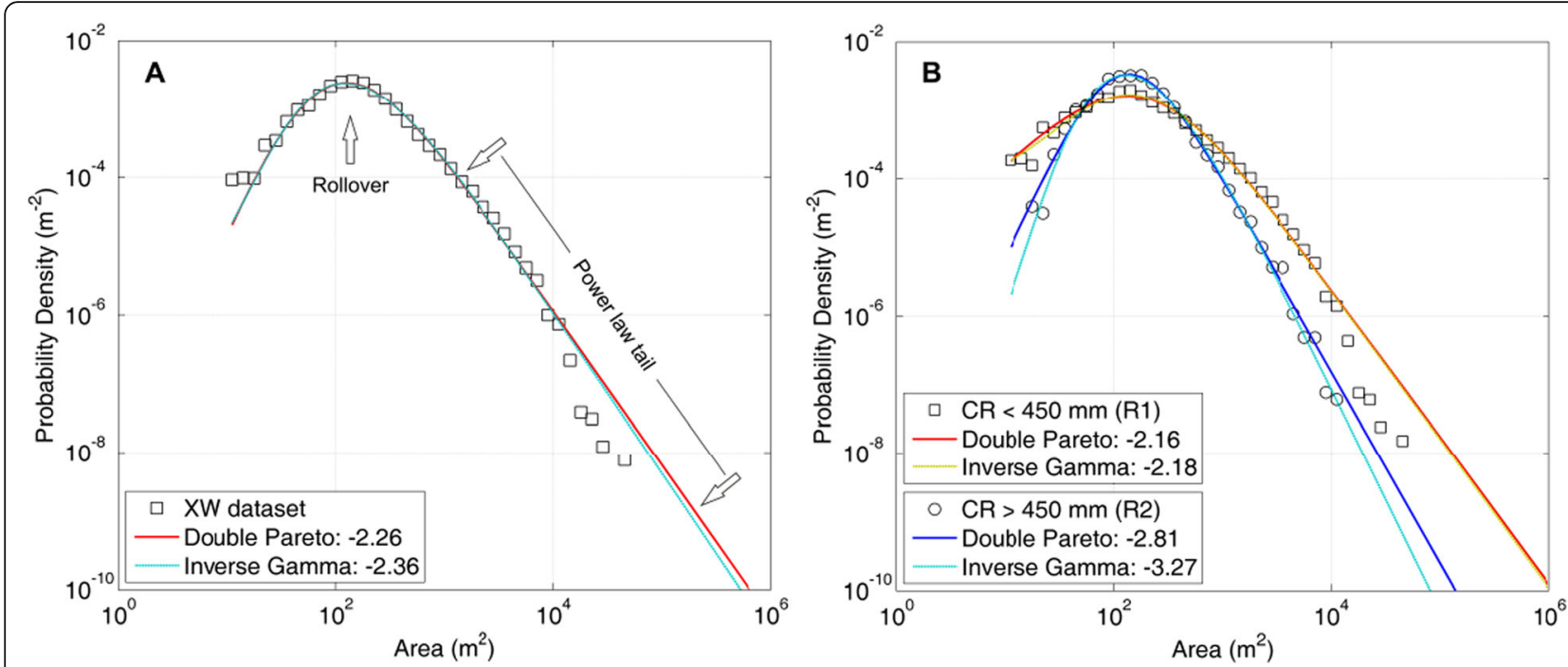

Fig. 2 Probability distributions of landslide area fitted to the XW dataset using the maximum likelihood estimation are shown. The scattered open squares and circles showing the empirical data represent the probability densities estimated based on histogram with logarithmic bins. Numerical values indicate the scaling exponents of the fitted power law tails. a The entire XW dataset. $\mathbf{b}$ The two subsets of the XW dataset identified according to cumulative rainfall

If we take $95 \%$ as a rule of thumb (Fig. 3e, f), the minimum sample size required for reliable parameter estimation can be inferred. However, finding a threshold universally applicable is unrealistic. It is related to not only the distribution function but also the theoretical parameters. Numerical experiments show that larger theoretical parameters (absolute values) require larger sample size to guarantee a high probability of low relative error. Nevertheless, Fig. 3 shows that 6,000 is a roughly safe choice for most landslide datasets. In view of this standard, utilizations of the parameters estimated based on small sample size, for example less than 1,000 (Fiorucci et al. 2011; Ghosh et al. 2012; Regmi et al. 2014), especially for quantitative use (Larsen and Montgomery 2012; Tsai et al. 2013), should be

Table 1 Statistical characteristics of landslide dataset and subsets

\begin{tabular}{|c|c|c|c|c|c|c|c|c|}
\hline \multirow[t]{2}{*}{ Dataset $^{a}$} & \multirow[t]{2}{*}{$N^{b}$} & \multicolumn{3}{|c|}{ Area $^{c}\left(m^{2}\right)$} & \multicolumn{2}{|c|}{ Double Pareto } & \multicolumn{2}{|c|}{ Inverse Gamma } \\
\hline & & Min & Max & $\overline{\text { Mean }}$ & $\gamma^{d}$ & $R^{\mathrm{e}}\left(\mathrm{m}^{2}\right)$ & $\gamma$ & $R\left(\mathrm{~m}^{2}\right)$ \\
\hline XW & 12,524 & 8 & 48,846 & 695 & -2.26 & 124.63 & -2.36 & 21.57 \\
\hline R1 & 6,297 & 8 & 48,846 & 1,021 & -2.16 & 133.82 & -2.18 & 137.85 \\
\hline R2 & 6,227 & 18 & 10,224 & 365 & -2.81 & 136.33 & -3.27 & 128.49 \\
\hline
\end{tabular}

"Dataset: "XW" indicates the entire $X W$ dataset, "R1" and "R2" indicate subsets of the $X W$ dataset characterized by a cumulative rainfall of lower and higher than $450 \mathrm{~mm}$, respectively

${ }^{\mathrm{b}} \mathrm{N}$ : number of landslides in a dataset (sample size)

"Area: "Min", "Max" and "Mean" indicate the minimum, maximum and mean landslide area of a dataset respectively

${ }^{d} \gamma$ : scaling exponent of the power law tail of the landslide area probability distribution fitted using the maximum likelihood method

${ }^{\mathrm{e}} R$ : rollover of the landslide area probability distribution fitted using the maximum likelihood method cautious. We will specifically inspect how sample size affects the reliability of landslide erosion estimates in the discussion.

The shape of landslide size distribution is defined by all the parameters, although the power law tail and the rollover are the two most characteristic features. As both double Pareto function and Inverse Gamma function have three variables, a good estimation of one parameter cannot guarantee a good estimation of another parameter. Thus, it is necessary to inspect the correlations between the estimated parameters besides inspecting them separately. The correlations between the estimated power exponents, rollovers and average volumes for sample size 10,000 and 1,000 are presented in Fig. 4. Generally, larger power exponents (absolute values) come with larger rollovers, while the average volume is inversely proportional to the power exponent (absolute value) and the rollover. Although these trends are distinct, scattering is also obvious in the plots. The less scattered results of larger sample size suggests more reliable estimates.

\section{The statistical significance of comparing parameters}

The regions for accepting the null hypothesis that "the differences in parameters between two different subsets of the same landslide dataset are derived from stochastic processes" are shown in Figs. 5 and 6 for $\gamma$ and $R$, respectively. The significance level is 0.05 , and the differences in parameters are the results of the parameters of the subsets with larger sample size subtracting that of the subsets with smaller sample size. Narrower region of 

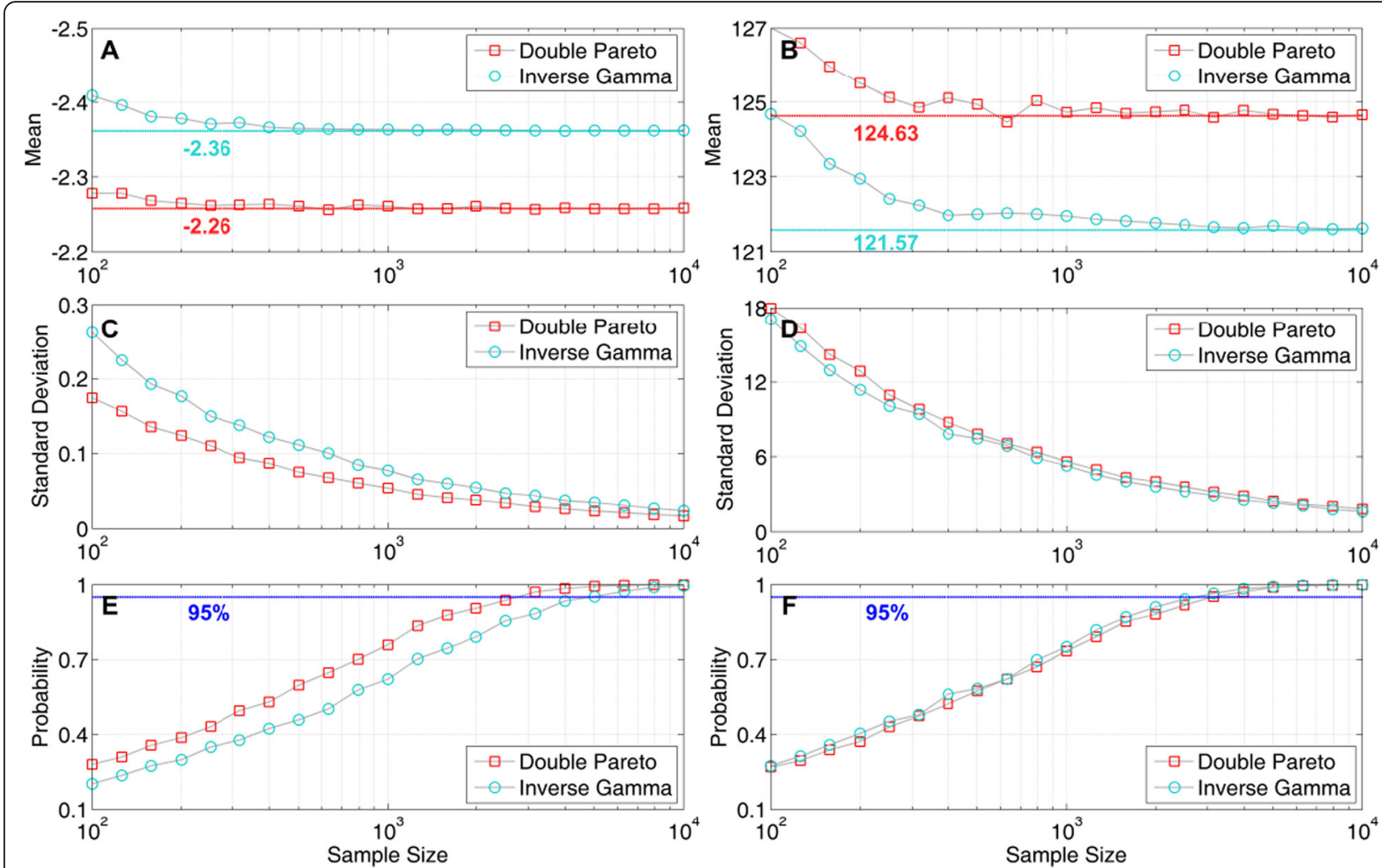

Fig. 3 The statistical characteristics of the estimated power exponents $(\gamma)$ and rollovers $(R)$ regarding different sample size are presented. a Mean of $\gamma$. $\mathbf{b}$ Mean of $R$. c Standard deviation of $\gamma$. d Standard deviation of $R$. e The probability that the estimated $\gamma$ has a relative error less than $5 \%$. f The probability that the estimated $R$ has a relative error less than $5 \%$. Horizontal lines in (a) and (b) indicate the theoretical values

acceptance means we have wider region of rejection in which we are confident in attributing the observed variances in parameters to physical constraints other than random processes.

It is shown that, regardless of distribution function, for both $\gamma$ and $R$, as sample size gets smaller or subdividing ratio gets larger, the region of acceptance gets wider (worse). It means smaller sample size and larger subdividing ratio expect larger differences in parameters to be observed for the sake of statistical significance. It also shows that the double Pareto distribution performs better on estimating the exponent (Fig. 5) while the Inverse Gamma distribution performs slightly better on estimating the rollover (Fig. 6). The regions of acceptance go beyond the range of figures for sample sizes less 250 if the subdividing ratio is large. This is because small sample size together with large subdividing ratio will yield unrealistic wide regions of acceptance. For example, with regard to a sample size of 100 and a subdividing ratio of 5 , the regions of acceptance for $\gamma$ estimated using the double Pareto distribution and the Inverse Gamma distribution are $[-14.69,13.75]$ and $[-18.22,15.24]$, respectively. Therefore, comparing the parameters of different subsets of a landslide dataset with an extreme small sample size, for instance less than 100 (Iwahashi et al. 2003), is practically statistically meaningless.

Numerical experiments show that, for the same sample size and subdividing ratio, larger parameters (absolute values) of the "mother dataset" yields wider regions of acceptance. Therefore, it is hard to find a universal standard for statistical significance. It is also hard to tell whether some published variances in parameters between different subsets is statistical significant or not, because either the MLE is not used (Chen 2009) or the information is not sufficient (Guzzetti et al. 2008). Nevertheless, test of statistical significance is highly recommended prior to physical interpretations of the variation of landslide size distribution between different subsets, especially for those with small sample size (Santangelo et al. 2013; Guns and Vanacker 2014). In the discussion, we will show that small sample size can cast a shadow on interpreting the physical constraints on landslide size distribution.

\section{Discussion}

The proposed statistical procedures in this paper is of potential use for exploiting landslide size distribution, including such as estimating landslide erosion rate, assessing 

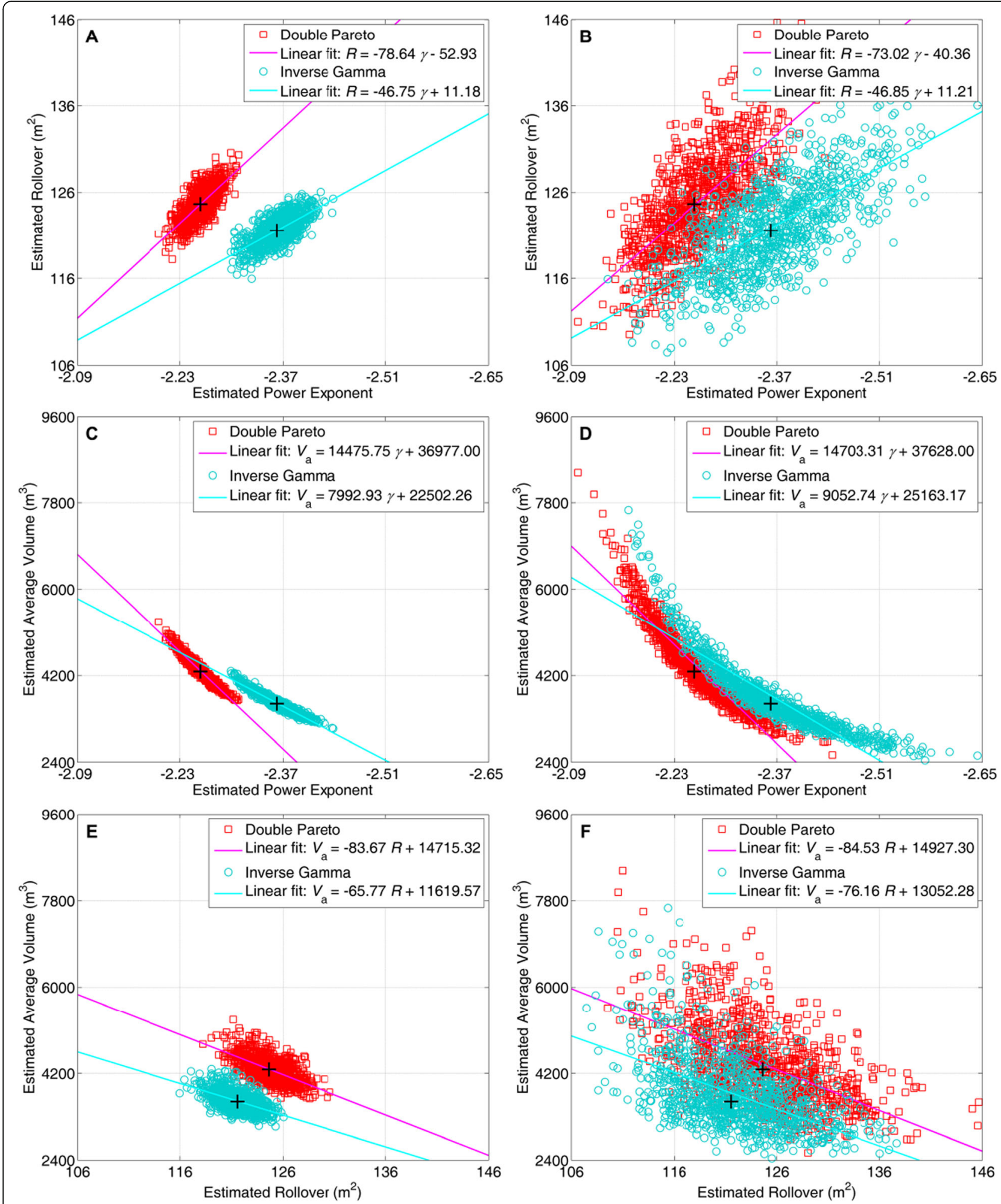

Fig. 4 The correlations between the estimated power exponents, rollovers and average volumes for sample size 10,000 (a, c, e) and 1,000 (b, d, f) are presented. Linear fits of scattered points are also provided 

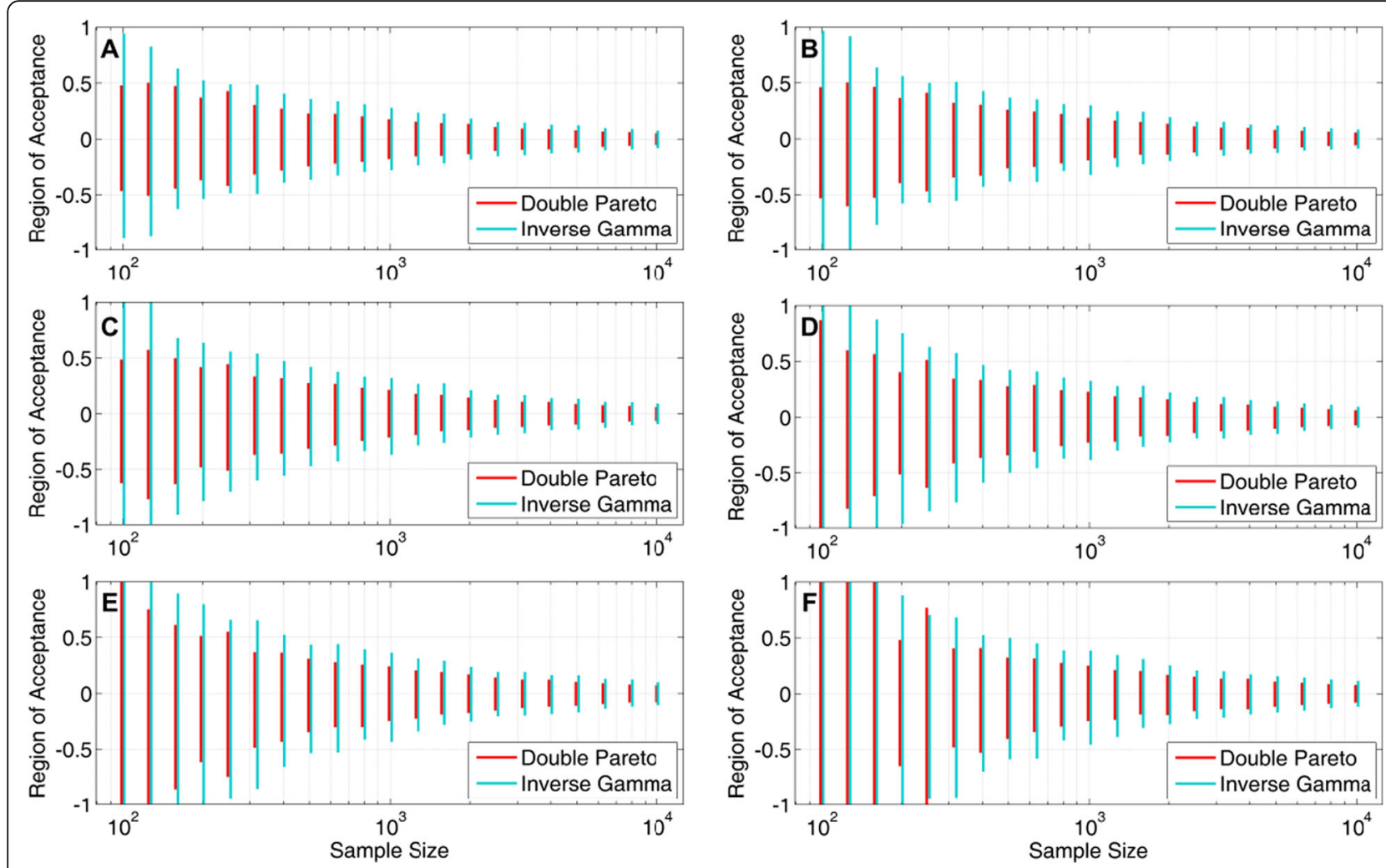

Fig. 5 The regions of acceptance for the null hypothesis that "the observed differences in power exponent ( $\gamma$ ) between two different subsets of the same dataset is derived from random processes" at a significance level of 0.05 are shown. $\mathbf{a}, \mathbf{b}, \mathbf{c}, \mathbf{d}, \mathbf{e}$ and $\mathbf{f}$ present the results for subdividing ratio $1: 1,2: 1,3: 1,4: 1,5: 1$ and $6: 1$, respectively. Please note that some of the regions of acceptance for sample sizes less than 250 go beyond the range of this figure, because small sample size together with large subdividing ratio will yield unrealistic wide regions of acceptance

landslide hazard and inspecting the physics of landslides. In this section, the effect of sample size on estimating landslide erosion rate is specifically discussed, and an example is presented to show that sample size can affect the confidence in attributing the variation of landslide size distribution to the spatial heterogeneity of rainfall intensity.

\section{The estimation of landslide erosion rate}

The statistical characteristics of the estimated average volume $\left(V_{\mathrm{a}}\right)$ with respect to each sample size are presented in Fig. 7. Similar to the power exponent and rollover, as sample size gets smaller, the mean of the estimated $V_{\mathrm{a}}$ gets more deviated from the theoretical value (Fig. 7a) while the standard deviation gets larger (Fig. 7b). However, the potential error of volume estimation is more significant compared with that of the power exponent and rollover. This can be seen from the probability that the estimated value has a relative error less than $5 \%$ (Fig. 7c). A sample size of 6,000 can only guarantee a probability of about $50 \%$ that the estimated $V_{\mathrm{a}}$ has a relative error less than $5 \%$. The relative error of the estimated $V_{\mathrm{a}}$ that has a probability equal to $50 \%$ is also calculated (Fig. 7d). It shows that, for the double Pareto distribution and Inverse Gamma distribution respectively, when sample size gets around 150 and 350, there will be half a chance that the estimated $V_{\mathrm{a}}$ has a relative error of $50 \%$. The double Pareto distribution performs relatively better on estimating the average volume than the Inverse Gamma distribution. However, even for the double Pareto distribution, there will be a probability of $50 \%$ that the estimated $V_{\mathrm{a}}$ has a relative error near $100 \%$ if the sample size is 100 (Fig. 7d). As landslide erosion rate is positively proportional to average landslide volume, the error of estimating $V_{\mathrm{a}}$ will directly bring the same error to the estimation of landslide erosion rate. In similar way, the potential error of assessing landslide hazard caused by insufficient sample size can be estimated, given the relationship between landslide size and landslide intensity is provided.

\section{The variation of landslide size distribution with rainfall intensity}

The significance of the observed variances in $\gamma$ and $R$ between the two subsets ( $R 1$ and $R 2)$ of the $X W$ dataset (Table 1) had been tested. We randomly subdivide the 

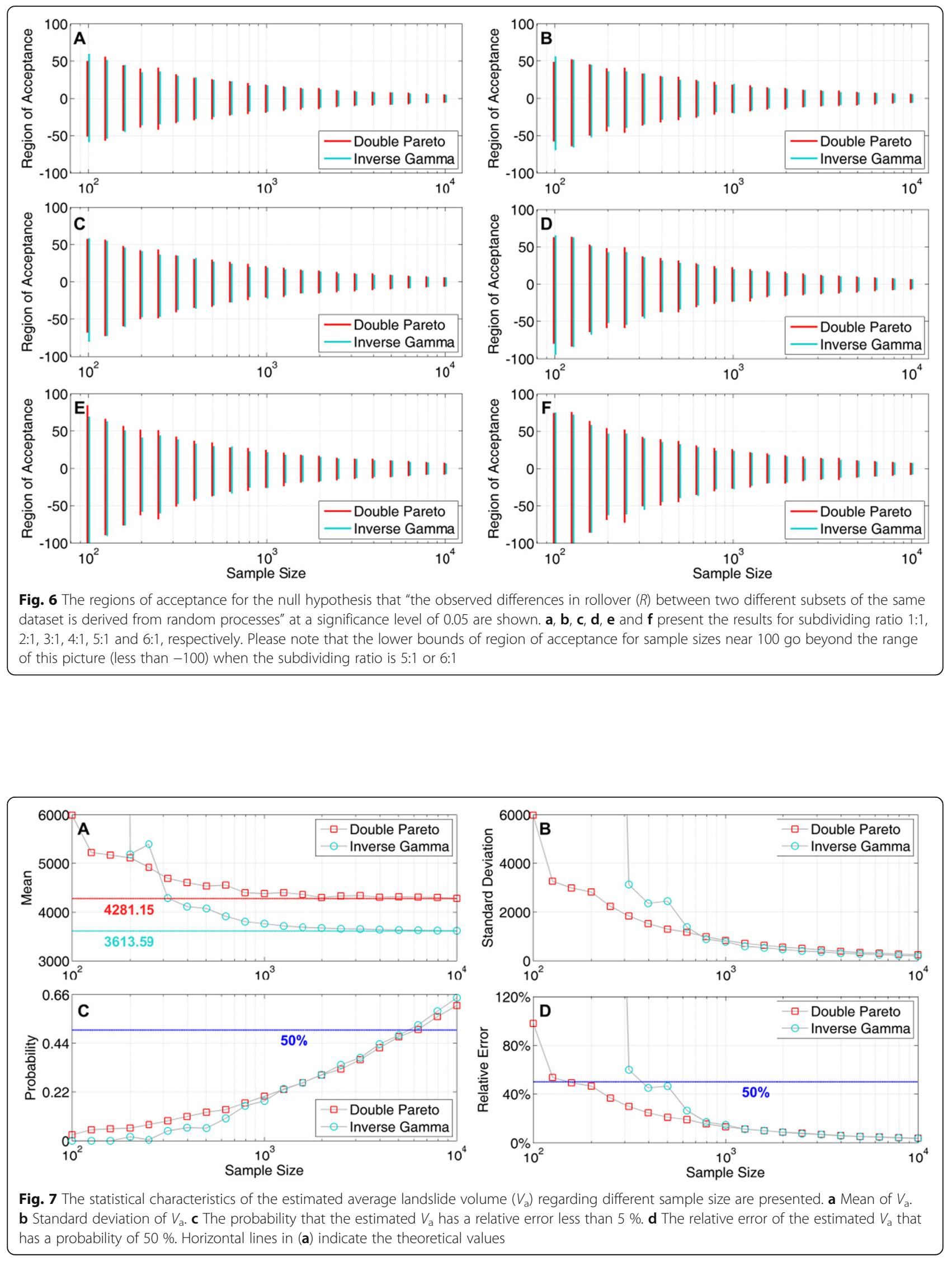
12,524 landslides into two subsets with 6,297 and 6,227 landslides respectively for 1,000 times. The results show that, all the differences in parameters observed between the $R 1$ and $R 2$ subsets is statistical significant at a significant level of 0.05, except for the difference in rollover estimated using the double Pareto distribution $(-2.51)$, which has a significant level of about 0.21 . Therefore, from the conservative point of view, we attribute the observed variances in rollover to random processes but suggest a physical explanation for the variances in power exponent.

We find that larger cumulative rainfall produces a steeper power law tail of the landslide area distribution (Fig. 2b). This is opposite to the previously reported result that the power law tail becomes flatter with an increase of the cumulative rainfall (Chen 2009). The variation of power exponent with rainfall intensity is essential because it concerns the problem whether increased rainfall intensity will increase the relative proportion of small size landslides or large size landslides. The explanation of this disagreement goes beyond the scope of this paper. Instead, we suggest a test of significance prior to physical interpretation. However, the statistical significance of the result published by Chen (2009) cannot be exactly told since the MLE was not used. Nevertheless, a variance of power exponent 0.27 is obtained by subdividing a landslide dataset with a sample size less than 600 . This result falls into the region of acceptance according to our numerical experiments (Fig. 4). Therefore, from a statistical point of view, there may be no adequate confidence to exclude the possibility that the variance of power exponent with rainfall intensity observed in Chen (2009) is due to random processes.

\section{Conclusions}

A series of numerical experiments were implemented in this paper to systematically quantify the effect of sample size on exploiting landslide area distribution. The results show that, as sample size gets smaller, both the reliability of the parameter estimation and the statistical significance of the variances in parameters observed between different subsets get worse. Therefore, quantitative analysis of landslide hazard and land surface erosion based on the statistics of landslide dataset with small sample size may be accompanied by considerable errors. Specifically, with a sample size of 100 , the relative error of the estimated landslide erosion rate that has a probability of $50 \%$ can approach $100 \%$. Furthermore, inconsistency was found regarding how the power exponent of landslide area distribution varies with rainfall intensity. Our numerical results suggest that the variance observed in a dataset with a small sample size may be not statistically significant. Although this study had focused on landslide area distribution and adopted the double Pareto distribution and the Inverse Gamma distribution, the presented procedures can be also used to quantify the potential effects of sample size regarding landslide volume distribution and other distribution functions.

Nevertheless, because the results of numerical simulations are affected by the statistical characteristics of the concerned landslide dataset, it is hard to find universally applicable criteria for adequate (large enough) sample size. A design for testing the potential effects of sample size on landslide size statistics but not a rule of thumb was proposed in this paper. It must be emphasized that, only larger sample size cannot guarantee reliable statistical results, a landslide dataset with physically representative sample distribution is usually a prerequisite.

\section{Acknowledgments}

This research was supported by National Natural Science Foundation of China (NO. 41525010, 41272354 and 41472282) and Research Foundation for Youth Scholars of IGSNRR, CAS. The authors also wish to thank Fujian Centre for Geological Environment Monitoring for SOPT images and relevant data. Mr. Zhiwei Wang is particularly appreciated for helping to prepare the landslide dataset in the Xiayang-Wangtai area.

\section{Authors' contributions}

LP designed the study, carried out the statistical analysis and drafted the manuscript. HX conceived of the study, and participated in its design and coordination and helped to draft the manuscript. YM participated in the statistical analysis. All authors read and approved the final manuscript.

\section{Competing interests}

The authors declare that they have no competing interests.

Received: 2 July 2016 Accepted: 4 October 2016

Published online: 23 October 2016

\section{References}

Alvioli, M., F. Guzzetti, and M. Rossi. 2014. Scaling properties of rainfall-induced landslides predicted by a physically based model. Geomorphology 213: 38-47. Brunetti, M.T., F. Guzzetti, and M. Rossi. 2009. Probability distributions of landslide volumes. Nonlinear Proc Geophys 16(2): 179-188.

Caers, J., J. Beirlant, and M.A. Maes. 1999. Statistics for modelling heavy tailed distributions in geology: Part I. Methodology. Mathematical Geoscience 31(4): 391-410.

Che, V.B., M. Kervyn, G.G.J. Ernst, P. Trefois, S. Ayonghe, P. Jacobs, E. van Ranst, and C.E. Suh. 2011. Systematic documentation of landslide events in Limbe area (Mt Cameroon Volcano, SW Cameroon): geometry, controlling, and triggering factors. Natural Hazards 59(1): 47-74.

Chen, C.Y. 2009. Sedimentary impacts from landslides in the Tachia River Basin, Taiwan. Geomorphology 105(3-4): 355-365.

Chen, C.C., L. Telesca, C.T. Lee, and Y.S. Su. 2011. Statistical physics of landslides: New paradigm. EPL 95(4): 49001

Cheng, Q.M. 2008. Non-linear theory and power-Law models for information integration and mineral resources quantitative assessments. Mathematical Geoscience 40: 503-532.

Clauset, A., C.R. Shalizi, and M.E.J. Newman. 2009. Power-law distributions in empirical data. SIAM Review 51(4): 661-703.

Fiorucci, F., M. Cardinali, R. Carlà, M. Rossi, A.C. Mondini, L. Santurri, F. Ardizzone, and F. Guzzetti. 2011. Seasonal landslide mapping and estimation of landslide mobilization rates using aerial and satellite images. Geomorphology 129(1-2): 59-70.

Frattini, P., and G.B. Crosta. 2013. The role of material properties and landscape morphology on landslide size distributions. Earth and Planetary Science Letters 361: 310-319.

Ghosh, S., C.J. van Westen, E.J.M. Carranza, V.G. Jetten, M. Cardinali, M. Rossi, and F. Guzzetti. 2012. Generating event-based landslide maps in a data-scarce Himalayan environment for estimating temporal and magnitude probabilities. Engineering Geology 128: 49-62. 
Guns, M., and V. Vanacker. 2014. Shifts in landslide frequency-area distribution after forest conversion in the tropical Andes. Anthropocene 6: 75-85.

Guzzetti, F., P. Reichenbach, M. Cardinali, M. Galli, and F. Ardizzone. 2005. Probabilistic landslide hazard assessment at the basin scale. Geomorphology 72(1-4): 272-299.

Guzzetti, F., F. Ardizzone, M. Cardinali, M. Galli, P. Reichenbach, and M. Rossi. 2008. Distribution of landslides in the Upper Tiber River basin, central Italy. Geomorphology 96(1-2): 105-122.

Guzzetti, F., F. Ardizzone, M. Cardinali, M. Rossi, and D. Valigi. 2009. Landslide volumes and landslide mobilization rates in Umbria, central Italy. Earth and Planetary Science Letters 279(3-4): 222-229.

Hovius, N., C.P. Stark, and P.A. Allen. 1997. Sediment flux from a mountain belt derived by landslide mapping. Geology 25(3): 231-234.

Hungr, O., S.G. Evans, and J. Hazzard. 1999. Magnitude and frequency of rock falls and rock slides along the main transportation corridors of southwestern British Columbia. Canadian Geotechnical Journal 36(2): 224-238.

Issler, D., F.V. De Blasio, A. Elverhøi, P. Bryn, and R. Lien. 2005. Scaling behaviour of clay-rich submarine debris flows. Mar Petrol Geol 22(1-2): 187-194.

Iwahashi, J., S. Watanabe, and T. Furuya. 2003. Mean slope-angle frequency distribution and size frequency distribution of landslide masses in Higashikubiki area, Japan. Geomorphology 50(4): 349-364.

Katz, O., and E. Aharonov. 2006. Landslides in vibrating sand box: What controls types of slope failure and frequency magnitude relations? Earth and Planetary Science Letters 247(3-4): 280-294.

Klar, A., E. Aharonov, B. Kalderon-Asael, and O. Katz. 2011. Analytical and observational relations between landslide volume and surface area. Journal of Geophysical Research 116(F02), F02001.

Kolyukhin, D., and J. Tveranger. 2014. Statistical analysis of fracture-length distribution sampled under the truncation and censoring effects. Mathematical Geoscience 46: 733-746.

Korup, O., T. Görüm, and Y. Hayakawa. 2012. Without power? Landslide inventories in the face of climate change. Earth Surf Proc Land 37(1): 92-99.

Larsen, I.J., and D.R. Montgomery. 2012. Landslide erosion coupled to tectonics and river incision. Nature Geoscience 5(7): 468-473.

Larsen, I.J., D.R. Montgomery, and O. Korup. 2010. Landslide erosion controlled by hillslope material. Nature Geoscience 3(4): 247-251.

Lazzeroni, L.C., and A. Ray. 2012. The cost of large numbers of hypothesis tests on power, effect size and sample size. Molecular Psychiatry 17(1): 108-114.

Lehmann, P., and D. Or. 2012. Hydromechanical triggering of landslides: From progressive local failures to mass release. Water Resources Research 48(3), W03535.

Li, L.P., H.X. Lan, and Y.M. Wu. 2012. Comment on "Statistical physics of landslides: New paradigm" by Chen C.-c. et al. EPL 100(2): 29001.

Li, L.P., H.X. Lan, and Y.M. Wu. 2014. The volume-to-surface-area ratio constrains the rollover of the power law distribution for landslide size. Eur Phys J Plus 129(5): 89.

Mackey, B.H., and J.J. Roering. 2011. Sediment yield, spatial characteristics, and the long-term evolution of active earthflows determined from airborne LiDAR and historical aerial photographs, Eel River, California. Geological Society of America Bulletin 123(7-8): 1560-1576.

Malamud, B.D., D.L. Turcotte, F. Guzzetti, and P. Reichenbach. 2004. Landslide inventories and their statistical properties. Earth Surf Proc Land 29(6): 687-711.

Montgomery, D.R., K. Sullivan, and H.M. Greenberg. 1998. Regional test of a model for shallow landsliding. Hydrological Processes 12(6): 943-955.

Pelletier, J.D., B.D. Malamud, T. Blodgett, and D.L. Turcotte. 1997. Scale-invariance of soil moisture variability and its implications for the frequency-size distribution of landslides. Engineering Geology 48(3-4): 255-268.

Pinto, C.M.A., A. Mendes Lopes, and J.A. Tenreiro Machado. 2012. A review of power laws in real life phenomena. Commun Nonlinear Sci Numer Simuln 17(9): 3558-3578.

Regmi, N.R., J.R. Giardino, and J.D. Vitek. 2014. Characteristics of landslides in western Colorado, USA. Landslides 11(4): 589-603.

Santangelo, M., D. Gioia, M. Cardinali, F. Guzzetti, and M. Schiattarella. 2013. Interplay between mass movement and fluvial network organization: An example from southern Apennines, Italy. Geomorphology 188: 54-67.

Stark, C.P., and F. Guzzetti. 2009. Landslide rupture and the probability distribution of mobilized debris volumes. Journal of Geophysical Research 114: F00A02.

Stark, C.P., and N. Hovius. 2001. The characterization of landslide size distributions. Geophysical Research Letters 28(6): 1091-1094.

ten Brink, U.S., R. Barkan, B.D. Andrews, and J.D. Chaytor. 2009. Size distributions and failure initiation of submarine and subaerial landslides. Earth and Planetary Science Letters 287(1-2): 31-42.

Tsai, Z.X., G.J.Y. You, H.Y. Lee, and Y.J. Chiu. 2013. Modeling the sediment yield from landslides in the Shihmen Reservoir watershed, Taiwan. Earth Surf Proc Land 38(7): 661-674.

\section{Submit your manuscript to a SpringerOpen ${ }^{\mathcal{O}}$ journal and benefit from:}

- Convenient online submission

- Rigorous peer review

- Immediate publication on acceptance

- Open access: articles freely available online

- High visibility within the field

- Retaining the copyright to your article

Submit your next manuscript at springeropen.com 